\title{
Patients of Myelodysplastic Syndrome with Mild/ Moderate Myelofibrosis and a Monosomal Karyotype are Independently Associated with an Adverse Prognosis: Long-Term Follow-Up Data
}

This article was published in the following Dove Press journal:

Cancer Management and Research

\author{
Na Wang $\mathbb{D}^{1-3}$ \\ Hongzhi $\mathrm{Xu}^{1,2}$ \\ Qing $\mathrm{Li}^{1,2}$ \\ Xiaosheng Fang (D) ${ }^{1,2}$ \\ Jie Liu $\mathbb{D}^{1,2}$ \\ Xiaohui Sui $\mathbb{D}^{1,2}$ \\ Lingyan Zhang ${ }^{1,2}$ \\ Yujie Jiang ${ }^{1,2}$ \\ Xin Wang (D) ${ }^{1-5}$
}

'Department of Hematology, Shandong Provincial Hospital Affiliated to Shandong First Medical University, Jinan, Shandong 25002 I, People's Republic of China;

${ }^{2}$ Department of Hematology, Shandong Provincial Hospital, Cheeloo College of Medicine, Shandong University, Jinan, Shandong 25002I, People's Republic of China; ${ }^{3}$ School of Medicine, Shandong University, Jinan, Shandong 250012 People's Republic of China; ${ }^{4}$ Shandong Provincial Engineering Research Center of Lymphoma, Jinan, Shandong 25002 I, People's Republic of China; ${ }^{5} \mathrm{National}$ Clinical Research Center for Hematologic Diseases, Jinan, Shandong 25002I, People's Republic of China
Correspondence: Xin Wang Department of Hematology, Shandong Provincial Hospital, Cheeloo College of Medicine, Shandong University, No. 324 Jingwu Road, Jinan, Shandong 25002I, People's Republic of China

Tel +86I3I560I 2606

Fax +86-53I-87068707

Email xinw@sdu.edu.cn
Purpose: The aim of our study was to evaluate the clinical characteristics of myelodysplastic syndrome (MDS) patients with concomitant mild-to-moderate myelofibrosis (MF), and to assess its independent prognostic role in MDS patients diagnosed by World Health Organization 2016 classification (WHO2016C) with long-term follow-up.

Patients and Methods: A total of 157 patients with primary MDS associated with or without MF were examined retrospectively with long-term follow-up. MF graded as MF-1/MF-2 was defined as "mild/moderate". Cytogenetics testing and fluorescence in situ hybridization (FISH) were also conducted in all MDS patients.

Results: Thirty-four (21.7\%) of 157 MDS patients had MF. Also, 24 (15.3\%) MDS patients based on WHO2016 criteria were defined as MF-1 and $10(6.4 \%)$ as MF-2. MDS patients with MF-1/2 had a higher prevalence of death $(p=0.002)$, leukemic progression $(p=0.013)$, O blood type $(p=0.039)$ as well as less hypercellular proliferation $(p<0.001)$ and less supportive treatment $(\mathrm{p}=0.003)$ compared with those without mild/moderate $\mathrm{MF}$. Cytogenetics testing did not show a significant difference between MDS patients with and without MF. Multivariate analyses showed that MF (mild/moderate), a monosomal karyotype (MK) and \% bone-marrow blasts were independently associated with shorter overall survival (OS) and progression-free survival (PFS). Age was an independent indicator of the adverse OS of MDS patients. Compared with those without MF, MDS patients with mild/moderate MF were significantly associated with worse OS and PFS in MK-negative subgroups and relatively low-risk Revised International Prognostic Scoring System for Myelodysplastic Syndromes (IPSS-R) stratification in long-term follow-up.

Conclusion: Mild/moderate myelofibrosis and monosomal karyotype are independent indicators of a poor clinical outcome in MDS patients. In long-term follow-up, MDS with mild/ moderate MF can be a prognostic marker for MDS patients with a specific MK stratification and IPSS-R stratification.

Keywords: myelodysplastic syndromes, myelofibrosis, prognosis, cytogenetics

\section{Introduction}

Myelodysplastic syndrome (MDS) is characterized by a heterogeneous clonal hematologic neoplasm with dysplasia. MDS with myelofibrosis (MF) is seen in $10-20 \%$ of MDS patients. ${ }^{1}$ Bone marrow (BM) biopsy is essential for the diagnosis and evaluation of MDS patients. In general, moderate-to-severe myelofibrosis is associated with an adverse prognosis in patients with primary MDS. ${ }^{1-6}$ However, 
according to the World Health Organization 2016 classification (WHO2016C), ${ }^{7}$ severe MF (MF grade 3 [MF-3]) is thought to be rare in MDS patients and is more likely diagnosed as MDS/myeloproliferative neoplasms or primary MF. MF-1 or MF-2 are usually accompanied with primary MDS. According to WHO2016 classification, the clinical importance of mild-to-moderate BM fibrosis in MDS patients is not clear. Whether BM fibrosis is a risk factor in the prognosis of MDS patients stratified according to WHO2016C is not known. Patients with MDS with MF are usually studied from molecular aspects, but the aspects of cytogenetics (eg, monosomal karyotypes $[\mathrm{MKs}])$ are controversial. Furthermore, the long-term effect of MF has not been evaluated.

We carried out a retrospective study on the prevalence and importance of mild/moderate MF on MDS patients according to WHO2016C. We wished to evaluate the clinical characteristics of MDS with concomitant mild-tomoderate MF. We also wanted to assess the impact of cytogenetics at long-term follow-up.

\section{Patients and Methods}

\section{Ethical Approval of the Study Protocol}

The study was conducted following the Declaration of Helsinki 1975 and its later amendments. The study protocol was approved by the institutional ethics review board of Shandong Provincial Hospital Affiliated to Shandong First Medical University (Shandong, China). Written informed consent was obtained from all patients.

\section{Patients}

According to WHO2016C, 157 patients with primary MDS were recruited from Shandong Provincial Hospital Affiliated to Shandong First Medical University from 1 January 2011 to 31 December 2016. The inclusion criteria were: (i) patients had been admitted to the hematology department and diagnosed as having primary MDS; (ii) the BM had been aspirated and biopsied for the initial diagnosis and could be assessed clearly. The exclusion criteria were patients who had been diagnosed with a myeloproliferative neoplasm, myelodysplastic/myeloproliferative neoplasm, or had therapy-related MDS.

All patients were followed up by telephone and outpatient appointments until 31 December 2019. Clinical demographics, complete blood counts, BM-blast counts, other clinical data and follow-up were recorded meticulously. All information was reviewed by different researchers to avoid biases.

\section{BM Biopsy}

All BM biopsies were of length $1-1.5 \mathrm{~cm}$ and diameter $0.2 \mathrm{~cm}$. BM aspirates were fixed in formalin and embedded in paraffin. Sections were stained with hematoxylin and eosin for morphology studies. Paraffin sections were stained using the Gomori silver-impregnation method. BM aspirates also underwent Wright staining and Giemsa staining.

Fibrosis was assessed according to European consensus guidelines. ${ }^{8}$ Grade 0 (absence of fibrosis) was defined as "the presence of scattered linear reticulin with no intersections". Grade 1 (MF-1, mild fibrosis) was defined as the presence of "a loose network of reticulin with many intersections, especially in perivascular areas". Grade 2 (MF-2, moderate fibrosis) was defined as "the diffuse and dense increase in reticulin with extensive intersections, occasionally with only focal bundles of collagen and/or focal osteosclerosis". Grade 3 (MF-3, severe fibrosis) was defined as "a diffuse and dense increase in reticulin with extensive intersections with coarse bundles of collagen, often associated with significant osteosclerosis" ${ }^{\prime \prime}$ MF-1 and MF-2 were defined as mild/moderate fibrosis.

\section{Cytogenetics}

Cytogenetic testing was carried out and reported using standard methods in BM samples according to the 2013 International System for Human Cytogenetic Nomenclature. ${ }^{9}$ Twenty metaphases were evaluated. Fluorescence in situ hybridization (FISH) was also undertaken to confirm additional clonal cytogenetic abnormalities. Cytogenetic risk stratification was assigned according to the Revised International Prognostic Scoring System for Myelodysplastic Syndromes (IPSS-R): ${ }^{10}$ "low" risk containing a normal karyotype and isolated loss of Y; "high" risk containing a complex karyotype ( $\geq 3$ abnormalities), trisomy 8, and abnormalities of chromosome 7; "intermediate" risk containing all other abnormalities. ${ }^{10}$ Besides, complementary FISH for abnormalities of chromosomes 5, 7, 8, 20 and $\mathrm{Y}$ was undertaken in all patients as described previously. ${ }^{11}$ A "complex karyotype" was defined as the presence of $\geq 3$ chromosome abnormalities. A "MK" was defined as the presence of a single autosomal monosomy accompanied with $\geq 1$ structural abnormality or $\geq 2$ distinct autosomal chromosome monosomies. 


\section{Definitions}

All patients were followed up until death or 31 December 2019 by telephone, on the ward, or in outpatient clinics. "Overall survival" (OS) of patients with MDS was defined from the date of the diagnosis to the date of last contact with the patient or death. "Progression-free survival" (PFS) was defined from the date of the diagnosis to the date of leukemia transformation, relapse, death, or last contact with the patient without disease progression.

\section{Statistical Analyses}

Statistical analyses were carried out using SPSS v18.0 (IBM, Armonk, NY, USA) and Prism 7 (GraphPad, San Diego, CA, USA). Numerical variables are described by their median and range and Categorical variables are described by counts and frequencies. The differences between patients with or without MF were compared by the Pearson chi-square test or Fisher exact test for categorical variables, as appropriate. Differences in continuous variables were compared with the Mann-Whitney $U$-test for two groups. OS and PFS were estimated by the Kaplan-Meier method and compared by the Log rank test. The Cox regression model was employed for univariable analysis and multivariable analysis to identify the most independent prognostic factors for OS and PFS. $\mathrm{P}<0.05$ (two-tailed) was considered significant.

\section{Results}

\section{Clinical Characteristics of MDS Patients with MF}

A total of 157 MDS patients were enrolled (86 [54.6\%] men and 71 [45.4\%] women; median age at the time of diagnosis, 54 [range, 11-82] years). Thirty-four (21.7\%) of these 157 MDS patients had BM fibrosis (20 [58.8\%] men and $14[41.2 \%]$ women). According to the European consensus ${ }^{8}$ on BM fibrosis, 24 (15.3\%) MDS patients were diagnosed as MF-1 and 10 (6.4\%) patients were MF-2. The demographic and clinical characteristics of MDS patients with and without mild/moderate MF are listed in Table 1. MDS patients with $\mathrm{mild} /$ moderate MF had a higher prevalence of death $(52.8 \% \mathrm{vs}$ $82.4 \%, \mathrm{p}=0.002)$ and leukemic progression $(20.3 \%$ vs $41.2 \%$, $\mathrm{p}=0.013$ ) compared with MDS patients without MF. Besides, MDS patients with mild/moderate MF received more aggressive treatment (decitabine/chemotherapy/hematopoietic stemcell transplantation) $(p=0.003)$ than those without MF. More
Table I Characteristics of MDS Patients with or without Myelofibrosis

\begin{tabular}{|c|c|c|c|}
\hline \multirow[t]{2}{*}{ Characteristics } & \multicolumn{2}{|c|}{ Myelofibrosis (Number of Cases) } & \multirow[t]{2}{*}{$p$} \\
\hline & $\begin{array}{l}\text { Grade } 0 \\
(n=123, \%)\end{array}$ & $\begin{array}{l}\text { Grade I-2 } \\
(n=34, \%)\end{array}$ & \\
\hline Sex (male) & $66(53.7 \%)$ & $20(58.8 \%)$ & 0.59 \\
\hline Age $\geq 55$ years & $57(46.3 \%)$ & $20(58.8 \%)$ & 0.20 \\
\hline $\begin{array}{l}\text { WHO } 2016 \\
\text { classification } \\
\text { 5q- syndrome } \\
\text { SLD } \\
\text { MLD } \\
\text { EB-I } \\
\text { EB-2 }\end{array}$ & $\begin{array}{l}7(5.7 \%) \\
18(14.6 \%) \\
71(57.7 \%) \\
9(7.3 \%) \\
18(14.6 \%)\end{array}$ & $\begin{array}{l}0(0 \%) \\
4(11.8 \%) \\
18(52.9 \%) \\
6(17.6 \%) \\
6(17.6 \%)\end{array}$ & 0.30 \\
\hline $\begin{array}{l}\text { IPSS-R risk } \\
\text { Very low } \\
\text { Low } \\
\text { Intermediate } \\
\text { High } \\
\text { Very high }\end{array}$ & $\begin{array}{l}\text { I }(0.8 \%) \\
38(30.9 \%) \\
43(35.0 \%) \\
25(20.3 \%) \\
16(13.0 \%)\end{array}$ & $\begin{array}{l}0(0.0 \%) \\
5(14.7 \%) \\
14(41.2 \%) \\
8(23.5 \%) \\
7(20.6 \%)\end{array}$ & 0.32 \\
\hline $\begin{array}{l}\text { WBC, } \times 10^{9} / \mathrm{L} \text {, median } \\
\text { (range) }\end{array}$ & $2.7(0.4-23.5)$ & $2.2(0.6-48.0)$ & 0.24 \\
\hline $\begin{array}{l}\text { HGB, g/L, median } \\
\text { (range) }\end{array}$ & $67(29-145)$ & $59.5(29-102)$ & 0.10 \\
\hline $\begin{array}{l}\text { ANC, } \times 10^{9} / \mathrm{L}, \text { median } \\
\text { (range) }\end{array}$ & $1.1(0.01-21.3)$ & $1.0(0.1-32.7)$ & 0.78 \\
\hline $\begin{array}{l}\mathrm{PLT}, \times 10^{12} / \mathrm{L} \text {, median } \\
\text { (range) }\end{array}$ & $40(3-44 I)$ & $47.5(2-3246)$ & 0.70 \\
\hline BM blast, \% & $1.0(0.0-19.0)$ & $2.5(0.0-17.0)$ & 0.10 \\
\hline Death & $65(52.8 \%)$ & $28(82.4 \%)$ & $0.002 *$ \\
\hline $\begin{array}{l}\text { Leukemic } \\
\text { transformation }\end{array}$ & $25(20.3 \%)$ & $14(41.2 \%)$ & $0.013^{*}$ \\
\hline $\begin{array}{l}\text { Degree of BM } \\
\text { hyperplasia } \\
\text { Hypercellular } \\
\text { Hypocellular } \\
\text { Normal }\end{array}$ & $\begin{array}{l}20(16.3 \%) \\
65(52.8 \%) \\
38(30.9 \%)\end{array}$ & $\begin{array}{l}16(47.1 \%) \\
8(23.5 \%) \\
10(29.4 \%)\end{array}$ & $<0.001^{*}$ \\
\hline $\begin{array}{l}\text { Blood type } \\
\text { Unexamined } \\
\text { AB } \\
\text { A } \\
\text { B } \\
\text { O }\end{array}$ & $\begin{array}{l}40(32.5 \%) \\
10(8.1 \%) \\
25(20.3 \%) \\
33(22.8 \%) \\
20(16.3 \%)\end{array}$ & $\begin{array}{l}5(14.7 \%) \\
0(0 \%) \\
10(29.4 \%) \\
14(29.8 \%) \\
11(32.4 \%)\end{array}$ & $0.039 *$ \\
\hline $\begin{array}{l}\text { Therapy } \\
\text { Chemotherapy }\end{array}$ & $4(3.3 \%)$ & $3(8.8 \%)$ & \\
\hline
\end{tabular}

(Continued) 
Table I (Continued).

\begin{tabular}{|l|l|l|l|}
\hline \multirow{2}{*}{ Characteristics } & \multicolumn{2}{|l|}{ Myelofibrosis (Number of Cases) } & \multirow{2}{*}{$p$} \\
\cline { 2 - 3 } & $\begin{array}{l}\text { Grade 0 } \\
(\mathbf{n}=123, \%)\end{array}$ & $\begin{array}{l}\text { Grade I-2 } \\
(\mathbf{n}=34, \%)\end{array}$ & \\
\hline Decitabine & $12(9.8 \%)$ & $11(32.4 \%)$ & \\
HSCT & $7(5.7 \%)$ & $0(0 \%)$ & \multirow{2}{*}{$0.003 *$} \\
Supportive therapy & $100(81.3 \%)$ & $20(58.8 \%)$ & \\
\hline
\end{tabular}

Note: *Significant difference.

Abbreviations: $A N C$, absolute neutrophil count; $B M$, bone marrow; $E B$, excess blasts; HGB, hemoglobin; HSCT, hematopoietic stem cell transplantation; IPSS-R, International Prognostic Scoring System-Revised; MDS, myelodysplastic syndrome; MLD, multilineage dysplasia; PLT, platelet; SLD, single lineage dysplasia; WBC, white blood cell count; WHO, World Health Organization.

hypercellular proliferation was observed in MDS patients with mild/moderate MF (47.1\%) than those without MF (14.6\%) ( $\mathrm{p}<0.001)$. MDS patients with MF tended to possess type-O blood compared with MDS patients without MF (32.4\% vs. $16.3 \%, p=0.039)$. There was no significant difference in sex, age, WHO2016C, IPSS-R risk stratification, leukocyte count, absolute neutrophil count, hemoglobin level, thrombopenia, or an excess number of BM blasts in MDS patients with and without MF.

\section{Cytogenetics}

Cytogenetic analysis was successful in 155 (98.7\%) of 157 MDS patients. FISH was conducted in all MDS patients and provided additional information in 28 (17.8\%) MDS patients. Sixteen (47.1\%) of $34 \mathrm{MDS}$ patients with mild/ moderate MF had a compatible abnormal karyotype with 68 (55.3\%) of 123 MDS patients without MF. Compared with MDS patients without MF, the prevalence of an MK and a complex karyotype was comparable between patients with or without MF. The most frequently typical karyotype was +8 with a similar frequency $(26.5 \%$ vs $17.9 \%, p=0.33)$ in patients with and without myelofibrosis. Besides, abnormalities involving a $5 \mathrm{q}$ deletion in chromosomes in MDS patients without MF tended to have a higher prevalence compared with those in MDS patients with mild/moderate MF $(14.6 \%$ vs $2.9 \%, p=0.077)$. All other karyotypes in the FISH panel showed a comparable prevalence in MDS patients with and without MF (Table 2).

\section{Prognostic Impact of MF in MDS Patients}

Follow-up data were available for all MDS patients. The median OS of 157 patients was 38.9 (range, 0.8-108) months, and the median PFS was 35.5 (0.8-108) months. To identify independent prognostic factors, we undertook
Table 2 Cytogenetics of MDS Patients with or without Myelofibrosis

\begin{tabular}{|l|l|l|l|}
\hline \multirow{2}{*}{ Cytogenetics } & \multicolumn{2}{|l|}{$\begin{array}{l}\text { Myelofibrosis (Number of } \\
\text { Cases) }\end{array}$} & \multirow{2}{*}{} \\
\cline { 2 - 3 } & $\begin{array}{l}\text { Grade 0 } \\
\text { (n = I 23, \%) }\end{array}$ & $\begin{array}{l}\text { Grade I-2 } \\
\text { (n = 34, \%) }\end{array}$ & \\
\hline Abnormal & $68(55.3 \%)$ & $16(47.1 \%)$ & 0.40 \\
Monosomal karyotype & $11(8.9 \%)$ & $3(8.8 \%)$ & 1.00 \\
Complex karyotype & $8(6.5 \%)$ & $3(8.8 \%)$ & 0.71 \\
$5 q-$ & $18(14.6 \%)$ & $1(2.9 \%)$ & 0.077 \\
-5 & $6(4.9 \%)$ & $0(0 \%)$ & 0.34 \\
$7 q-$ & $9(7.3 \%)$ & $3(8.8 \%)$ & 0.72 \\
-7 & $14(11.4 \%)$ & $4(11.8 \%)$ & 1.00 \\
+8 & $22(17.9 \%)$ & $9(22.6 \%)$ & 0.27 \\
$20 q-$ & $20(16.3 \%)$ & $2(5.9 \%)$ & 0.17 \\
$-Y$ & $5(4.1 \%)$ & $0(0 \%)$ & 0.59 \\
\hline
\end{tabular}

univariate analyses using age, sex, WHO2016C, white blood cell count, hemoglobin level, absolute neutrophil count, platelet count, percentage of BM blasts, blood type, cytogenetic risk group of IPSS-R, BM cellularity, and MF as factors. We also undertook univariate analyses of common MDS alterations in cytogenetics $(-5, \operatorname{del}(5 q),-7, \operatorname{del}(7 q)$, del (20q), +8, -Y), MK, and complex karyotypic abnormalities. Univariate analyses showed the OS of MDS patients to be closely related to sex (hazard ratio [HR], 1.64; 95\% confidence interval (CI), 1.02-2.34), age (2.02, 1.33-3.06), WHO2016C (1.19, 1.00-1.42), MF (2.04, 1.30-3.18), \% BM blasts (1.13,1.10-1.17), MK (3.88, 2.13-7.05), complex karyotype $(5.19,2.67-10.09)$ and IPSS-R (2.00,1.63-2.44). Univariate analyses also showed the PFS of MDS patients to be closely related to sex (HR, 1.63; 95\% CI, 1.06-2.51), age (1.50, 0.98-2.28), WHO2016C (1.28, 1.08-1.53), MF (1.87, 1.18-2.99), \% BM blasts $(1.14,1.10-1.81)$, MK (3.46,1.90-6.29), complex karyotype (4.07, 2.12-7.85) and IPSS-R (2.13,1.72-2.63). The univariate analysis also showed that MDS patients with chromosome $(-5)$ or with $(-7)$ were related to the worse OS and PFS than those without them $(\mathrm{p}<0.001)$. Meanwhile, MDS patients with cytogenetics $(\operatorname{del}(5 q), \operatorname{del}(7 q), \operatorname{del}(20 q),+8,-Y)$ did not show any differences in OS and PFS, compared with those without corresponding cytogenetics. Considering their close correlation with IPSS-R and MK, we did not involve chromosome $(-7)$ and $(-5)$ as covariates in the multivariate model. The other factors we tested were not significant. Multivariate analyses showed that MF ( $\mathrm{p}=0.014 ; \mathrm{HR}, 1.77 ; 95 \% \mathrm{CI}$, $1.12-2.77)$, MK $(<0.001 ; 3.50 ; 1.90-6.46)$, \% BM blasts $(<0.001 ; 1.12 ; 1.08-1.16)$ and age at the diagnosis $(0.002$; 
Table 3 Cox Regression Analysis for Overall Survival in MDS Patients

\begin{tabular}{|l|l|l|l|l|l|l|}
\hline \multirow{2}{*}{ OS variables } & \multicolumn{2}{l|}{ Univariate Analysis } & \multicolumn{2}{l|}{ Multivariate Analysis } \\
\cline { 2 - 7 } & $\mathbf{P}$ & $\mathbf{H R}$ & $\mathbf{9 5 \%} \mathbf{C l}$ & $\mathbf{P}$ & $\mathbf{H R}$ \\
\hline Sex (male) & 0.040 & 1.54 & $1.02-2.34$ & 0.10 & $\mathbf{9 5 \%} \mathbf{C l}$ \\
Age (>55 years) & 0.001 & 2.02 & $1.33-3.06$ & $0.002^{*}$ & 1.02 & \\
WHO classification & 0.048 & 1.19 & $1.00-1.42$ & 0.24 & $1.01-1.04$ \\
Myelofibrosis & 0.002 & 2.04 & $1.30-3.18$ & $0.014^{*}$ & 1.77 & $1.12-2.77$ \\
BM blasts & $<0.001$ & 1.13 & $1.10-1.17$ & $<0.001^{*}$ & 1.12 & $1.08-1.16$ \\
Monosomal karyotype & $<0.001$ & 3.88 & $2.13-7.05$ & $<0.001^{*}$ & 3.50 & $1.90-6.46$ \\
Complex karyotype & $<0.001$ & 5.19 & $2.67-10.09$ & 0.28 & & \\
IPSS-R & $<0.001$ & 2.00 & $1.63-2.44$ & 0.068 & & \\
\hline
\end{tabular}

Note: *Significant difference.

Abbreviations: BM, bone marrow; $\mathrm{Cl}$, confidence interval; HR, hazard ratio; MDS, myelodysplastic syndrome; OS, overall survival; PFS, progression-free survival; WHO, World Health Organization.

Table 4 Cox Regression Analysis for Progression-Free Survival in MDS Patients

\begin{tabular}{|l|l|l|l|l|l|l|}
\hline \multirow{2}{*}{ PFS variables } & \multicolumn{2}{l|}{ Univariate Analysis } & \multicolumn{2}{l|}{ Multivariate Analysis } \\
\cline { 2 - 7 } & $\mathbf{P}$ & $\mathbf{H R}$ & $\mathbf{9 5 \%} \mathbf{~ I ~}$ & $\mathbf{P}$ & $\mathbf{H R}$ & $\mathbf{9 5 \%} \mathbf{C l}$ \\
\hline Sex (male) & 0.024 & 1.61 & $1.07-2.43$ & 0.067 & \\
Age (>55 years) & 0.009 & 1.73 & $1.15-2.60$ & 0.19 & \\
WHO classification & 0.017 & 1.23 & $1.04-1.45$ & 0.16 & \\
Myelofibrosis & 0.002 & 2.02 & $1.30-3.15$ & $0.003^{*}$ & 1.99 & 1.14 \\
BM blasts & $<0.001$ & 1.14 & $1.10-1.18$ & $<0.001^{*}$ & $1.27-3.11$ \\
Monosomal karyotype & $<0.001$ & 3.28 & $1.81-5.96$ & $<0.001^{*}$ & 3.05 & $1.10-1.19$ \\
Complex karyotype & $<0.001$ & 4.07 & $2.12-7.85$ & 0.41 & & $1.67-5.56$ \\
IPSS-R & $<0.001$ & 2.02 & $1.65-2.47$ & 0.17 & & \\
\hline
\end{tabular}

Note: *Significant difference.

Abbreviations: $\mathrm{BM}$, bone marrow; $\mathrm{Cl}$, confidence interval; HR, hazard ratio; MDS, myelodysplastic syndrome; OS, overall survival; PFS, progression-free survival; WHO, World Health Organization.

$1.02 ; 1.01-1.04)$ were independently associated with a shorter OS in MDS patients (Table 3). MF (mild/moderate) $(\mathrm{p}=0.003 ; \mathrm{HR}, 1.99 ; 95 \% \mathrm{CI}, 1.27-3.11)$, MK $(<0.001$; $3.05 ; 1.67-5.56)$ and $\%$ BM blasts $(<0.001 ; 1.14 ; 1.10-1.19)$ were also independent indicators for shorter PFS (Table 4).

MDS patients with mild/moderate MF had a significantly shorter OS (median, 17.7 vs 47.6 months, $\mathrm{p}=0.001)$ and PFS (13.5 vs 42.0 months, 0.002) compared with those without MF (Figures 1A and 2A). OS declined gradually according to MF severity in MDS patients. MDS patients with MF-1 had much shorter OS than those with MF-0 (median, 25.5 vs 47.6 months, $p=0.048$ ). OS of MDS patients with MF-2 was shorter than that of cases with MF-1 (median, 13.5 vs 25.5 months, $p=0.049$ ) (Figure 1B). PFS of MDS patients with MF-1 was shorter than that of patients with MF-0 (median, 25.5 vs 42.0 months, $p=0.047$ ). PFS of MDS patients with MF-2 was not significantly different from that of patients with MF-1 (median, 9.9 vs 25.5 months, $\mathrm{p}=0.076$ ) (Figure 2B).

\section{Outcome of MDS with MF in Subgroup Analyses with Long-Term Follow-Up}

We undertook subgroup analyses for OS and PFS in MDS patients with long-term follow-up based on IPSS-R risk stratification and MK, which also affected OS and PFS independently. Subgroup analyses showed that MDS patients with mild/moderate MF represented an inferior OS (median, 36.4 months vs not reach, $p=0.008$ ) compared with those without $\mathrm{MF}$ in the relatively low-risk group of IPSS-R (including very low, low, and intermediate risk) (Figure 1C). However, there was no significant difference in OS between patients with mild/moderate MF and those without MF in the relatively high-risk group (including high and very high risk) of IPSS$\mathrm{R}$ (Figure 1D). In the relatively low-risk group of IPSS-R, MDS patients with mild/moderate MF had shorter PFS (median, 36.4 months vs not reach, $p=0.009$ ) compared with those with MDS without MF. There was no significant difference between patients with mild/moderate MF and without MF in the relatively high-risk group of IPSS-R (Figure 2C and D). 
A

Overal survival $(n=157)$

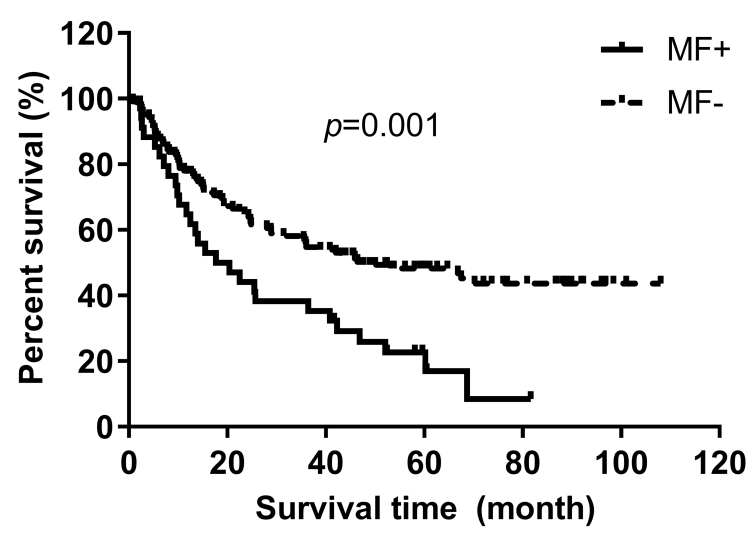

C

OS for relative low risk of IPSS-R $(n=101)$

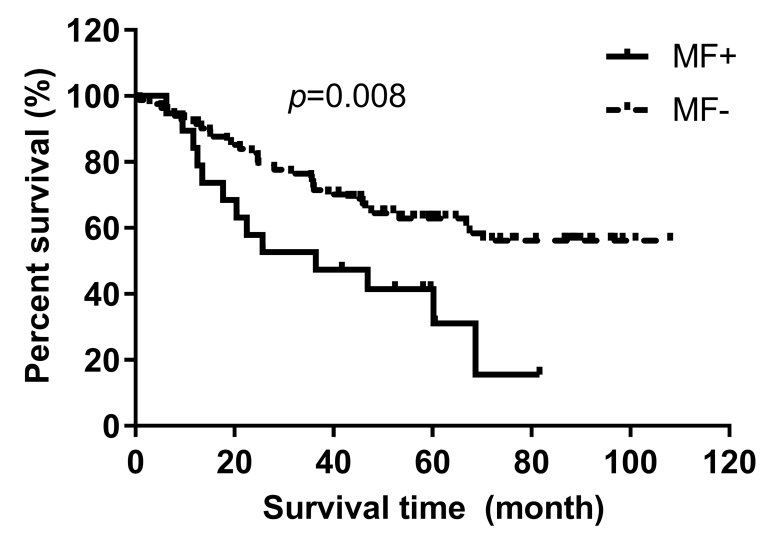

E

Overal survival of MK- group $(n=143)$

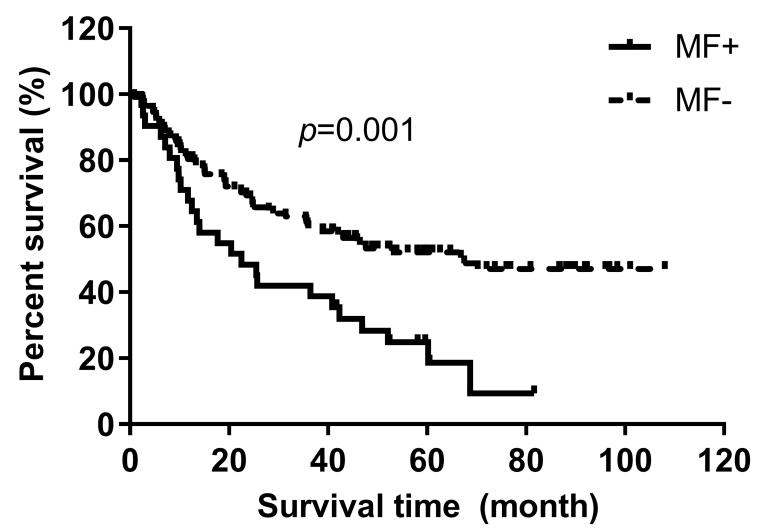

B

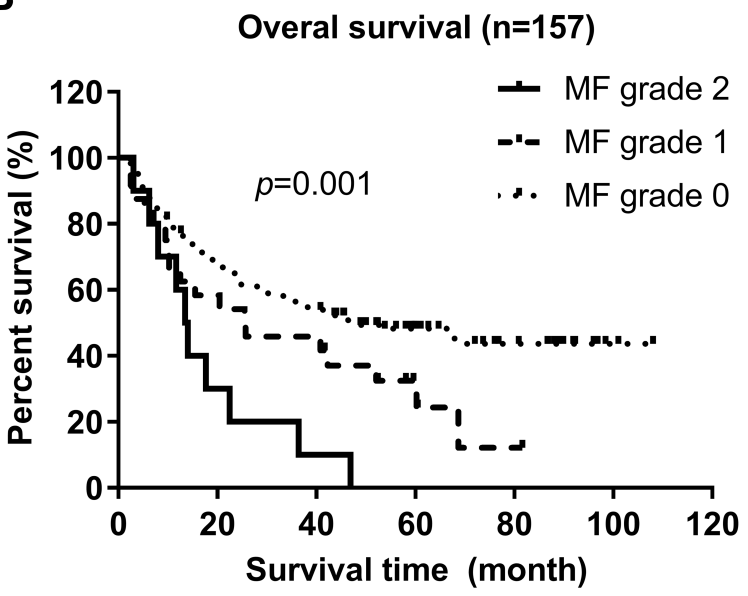

D

OS for high risk group of IPSS-R $(n=56)$

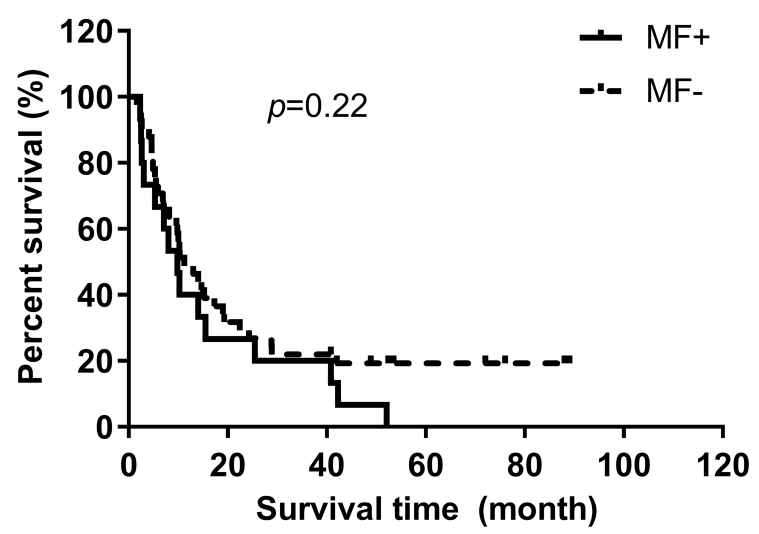

$\mathbf{F}$

Overall survival of MK+ group $(n=14)$

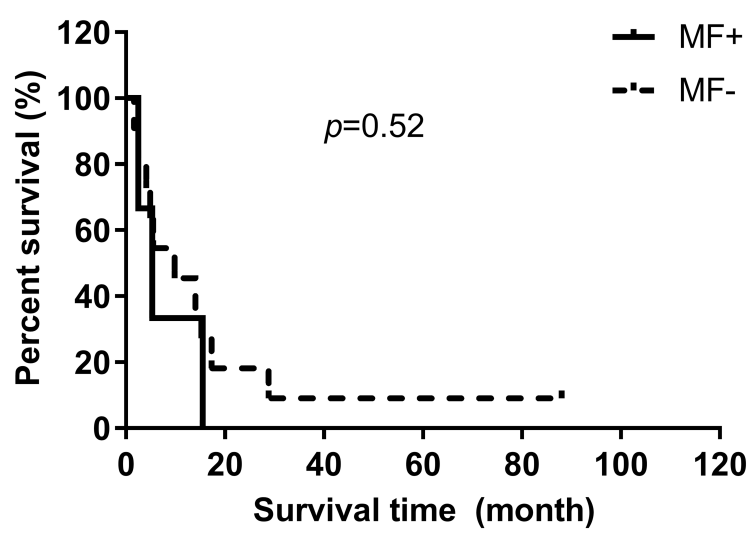

Figure I Comparison of overall survival (OS) of MDS patients with or without mild/moderate myelofibrosis (MF). (A) OS in MF+ and MF- MDS patients. (Log rank test, $P=$ $0.00 \mathrm{I}$ ). (B) OS of MDS patients according to MF grade (Log rank test,p $=0.00 \mathrm{I}$ ); OS of MDS patients with grade I vs grade 0 (Log rank test, $\mathrm{p}=0.048$ ); OS of MDS patients with grade 2 vs grade 0 (Log rank test, $p<0.00$ I); OS of MDS patients with grade 2 vs grade I (Log rank test, $p=0.05$ ). (C) OS of MDS patients with relatively lower IPSS-R risk with or without mild/moderate MF (Log rank test, $p=0.008)$. (D) OS of MDS patients with higher IPSS-R risk with or without mild/moderate MF. (Log rank test, $p=$ 0.22). (E) OS of MK- MDS patients with or without mild/moderate MF (Log rank test, $p=0.00 \mathrm{I})$. (F) OS of MK+ MDS patients with or without mild/moderate MF (Log rank test, $\mathrm{P}=0.52$ ).

Abbreviations: MDS, myelodysplastic syndrome; MF, myelofibrosis; MK, monosomal karyotype; OS, overall survival; PFS, progression-free survival. 
A

Progression free survival $(n=157)$

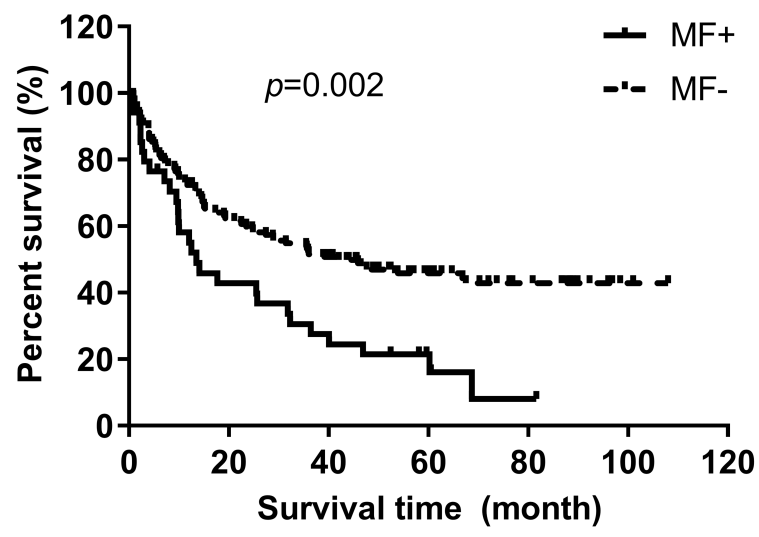

C

PFS for relative low risk of IPSS-R $(n=101)$

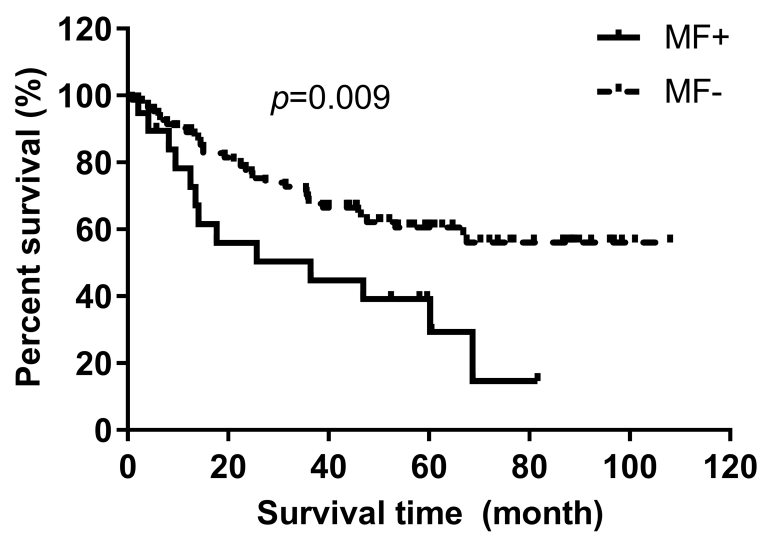

E

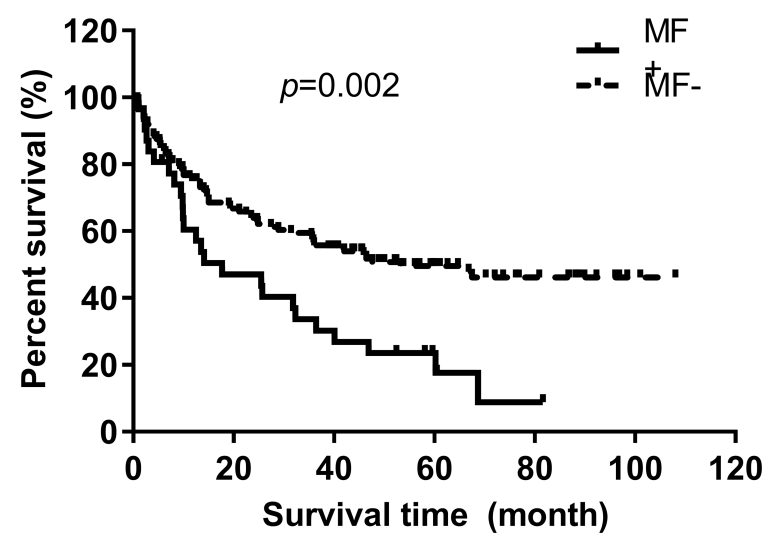

B

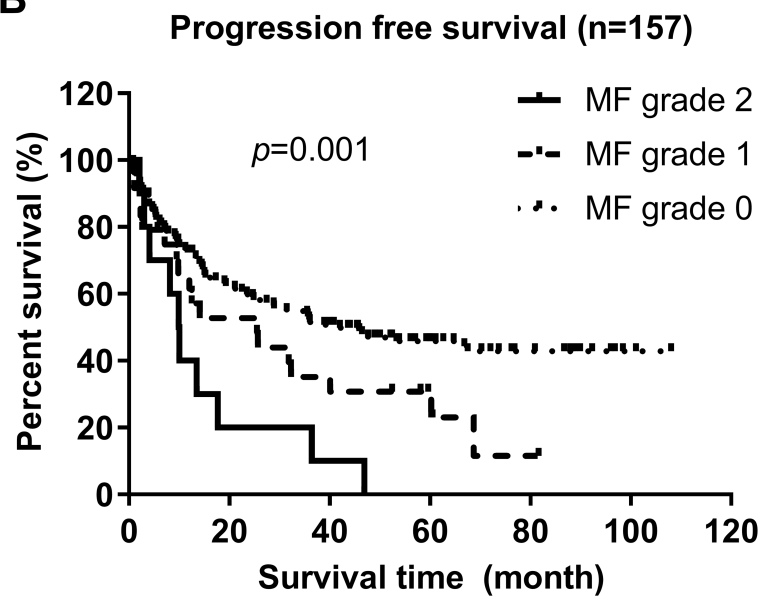

D

PFS for relative high risk of IPSS-R ( $n=56)$

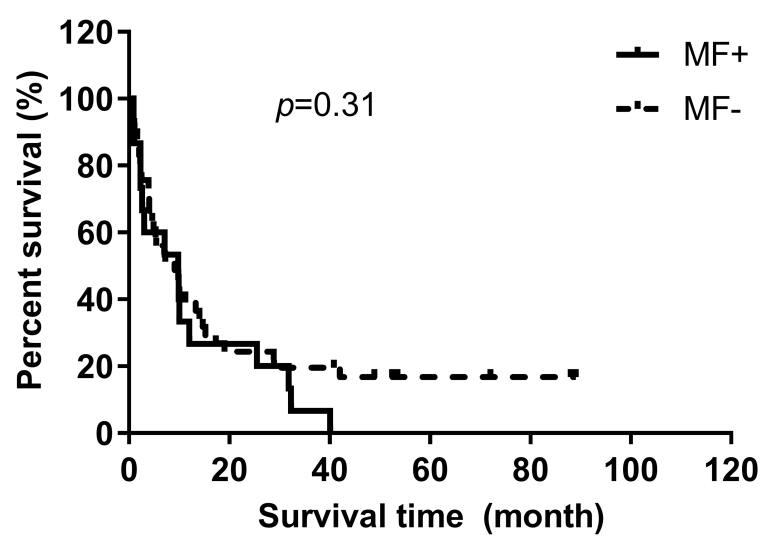

$\mathbf{F}$

PFS of MK+ group $(n=14)$

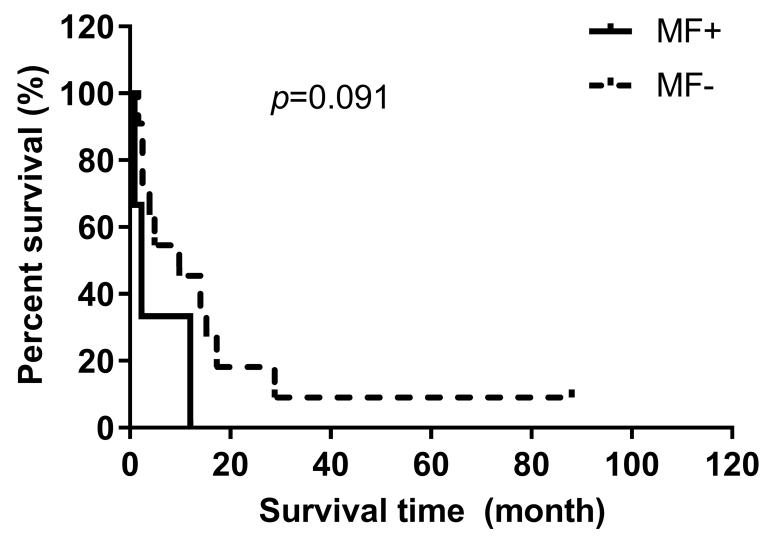

Figure 2 Comparison of progression-free survival (PFS) of MDS patients with or without mild/moderate myelofibrosis (MF). (A) PFS in MF+ and MF- MDS patients (Log rank test, $p=0.002$ ). (B) PFS of MDS patients according to MF grade (Log rank test, $P=0.00 \mathrm{I}$ ); PFS of MDS patients with grade I vs grade 0 (Log rank test, $P=0.047)$; PFS of MDS patients with grade 2 vs grade 0 (Log rank test, $p=0.00 \mathrm{I}$ ); PFS of MDS patients with grade 2 vs grade I (Log rank test, $p=0.076$ ). (C) PFS of MDS patients with relatively lower IPSS-R risk with or without mild/moderate MF (Log rank test, $p=0.009$ ). (D) PFS of MDS patients with higher IPSS-R risk with or without mild/moderate MF (Log rank test, $p=0.31$ ). (E) PFS of MK- MDS patients with or without mild/moderate MF (Log rank test, $p=0.002)$. (F) PFS of MK+ MDS patients with or without mild/ moderate MF (Log rank test, $p=0.091)$.

Abbreviations: MDS, myelodysplastic syndrome; MF, myelofibrosis; MK, monosomal karyotype; OS, overall survival; PFS, progression-free survival. 
According to the MK subgroup, MDS patients with mild/ moderate MF were associated significantly with worse OS (median, 22.5 vs 67.5 months, $\mathrm{p}=0.001$ ) and PFS (17.7 vs 53.3 months, 0.002) compared with those without MF in the MK-negative subgroup; however, they did not show a significant difference in OS or PFS compared with those without MF in the MK-positive subgroup (Figure 1E and F; Figure $2 \mathrm{E}$ and $\mathrm{F}$ ).

We also undertook subgroup analyses for OS and PFS in MDS patients with long-term follow-up based on supportive and non-supportive treatment (hematopoietic stem-cell transplantation, decitabine, chemotherapy). There were no differences in OS (median,13.9 vs 19.3 months, $\mathrm{p}=0.30$ ) or PFS (10.0 vs 13.3 months, 0.72$)$ between MDS patients with or without mild/moderate $\mathrm{MF}$ in the non-supportivetreatment subgroup. However, in the supportive-treatment subgroup, MDS patients with mild/moderate MF were associated significantly with worse OS (median, 25.5 vs 67.5 months, $\mathrm{p}=0.028$ ) and PFS (25.5 vs 67.5 months, 0.016) compared with those without MF (Figure 3).

\section{Discussion}

Severe MF is thought to be rare in MDS patients. Mild and moderate MF in MDS patients is associated with higher
A

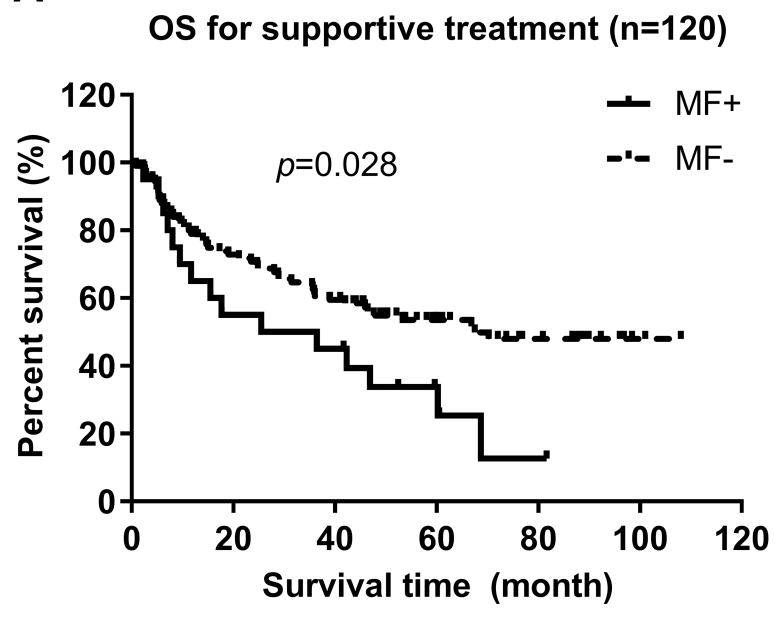

C OS of HSCT/Dec/CT treatment $(n=37)$

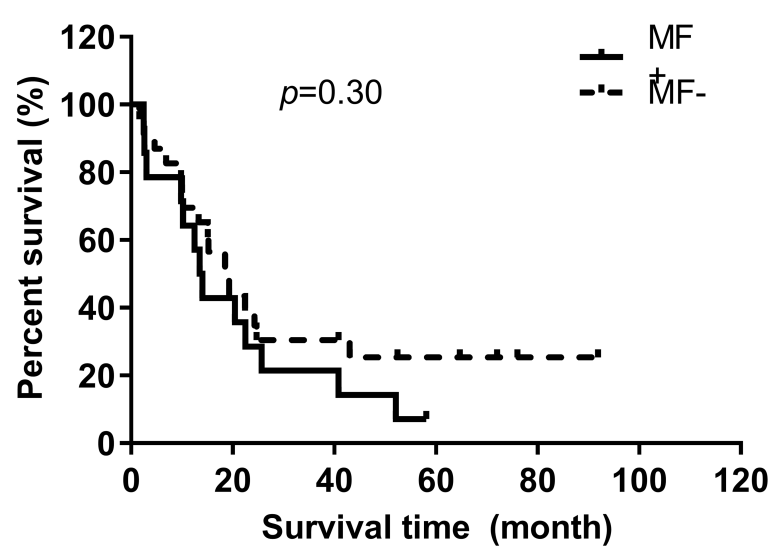

B

PFS for supportive treatment $(n=120)$

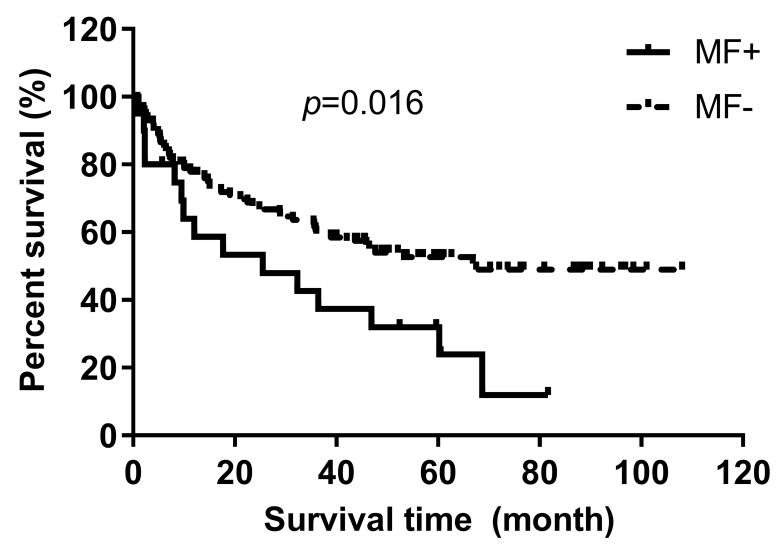

D PFS of HSCT/Dec/CT treatment $(n=37)$

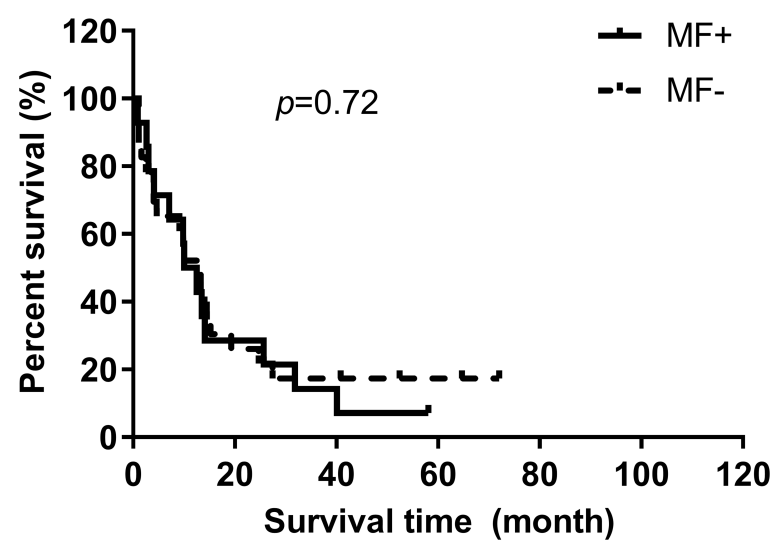

Figure 3 Comparison of overall survival (OS) and progression-free survival (PFS) of MDS patients with or without myelofibrosis (MF) according to different treatments. (A) OS of MDS patients with or without mild/moderate MF in the supportive-treatment subgroup (Log rank test, $P=0.028$ ). (B) PFS of MDS patients with or without mild/ moderate MF in the supportive-treatment subgroup (Log rank test, $\mathrm{p}=0.016$ ). (C) OS of MDS patients with or without mild/moderate MF in the HSCT/decitabine/ chemotherapy subgroup (Log rank test, $P=0.30$ ). (D) PFS of MDS patients with or without mild/moderate MF in the HSCT/decitabine/chemotherapy subgroup (Log rank test, $p=0.72$ ).

Abbreviations: HSCT, hematopoietic stem cell transplantation; MDS, myelodysplastic syndrome; MF, myelofibrosis; OS, overall survival; PFS, progression-free survival. 
mortality, leukemic progression, less hypercellular proliferation, O-type blood, and more aggressive treatment compared with that in patients without MF. Cytogenetics did not show a significant difference between MDS patients with and without MF. Furthermore, we showed that MF (mild and moderate) also had an adverse prognosis on MDS patients, as well as those with an MK and more BM blasts. Compared with those without MF, MDS patients with mild/moderate MF were also associated significantly with worse OS and PFS in the MK-negative subgroup, relatively low-risk group of IPSS-R and supportive treatment subgroup in long-term follow-up.

We showed that MF occurred at mild and moderate grades in $21.7 \%$ of patients at the time of the initial diagnosis. This prevalence is comparable with that reported previously $(10-20 \%) .^{2,5,6,12-14}$ Compared with other studies, ${ }^{1,5,13}$ mild/moderate MF in MDS patients was not associated with a lower platelet count and more severe anemia in our study. Some scholars have shown that MDS patients with MF have a lower prevalence of anemia $^{5}$ and lower platelet count compared with those without MF. ${ }^{1,13}$ The differences in results between studies are likely attributable to the evolving definition of MDS and distribution of enrolled patients.

Interestingly, compared with patients with MDS without MF, MDS patients with mild/moderate MF had type-O blood: this has not been reported previously. Jaff and colleague showed that significantly more patients with primary MF had group-B blood. ${ }^{15}$ Several scholars have reported that the $\mathrm{ABO}$ blood type might be related to some cancer types, such as cancer of the pancreas, ${ }^{16}$ and skin. ${ }^{17}$ It has been reported that compared with the type-O blood, the non-O blood type is associated with shorter OS for some cancer patients. ${ }^{16,18,19} A B O$ is located on chromosome $9 \mathrm{q} 34$ and related to some genetic-coding phenotypes. ${ }^{20}$ In the future, we will undertake studies to ascertain the differences in MDS patients with MF and O blood type and those with different blood types.

In our study, MDS with MF showed a similar percentage of BM blasts with those without MF. Fu and colleagues showed MDS with severe MF to be accompanied by higher numbers of BM blasts. ${ }^{13}$ However, our research subject focused on MDS patients with mild/moderate MF rather than those with severe MF. Besides, MDS patients with mild/moderate MF in our study tend to have an increased rate of leukemic evolution and death consistent with MDS patients with severe MF reported by Della Porta and colleagues. ${ }^{5}$
We observed a much higher prevalence of abnormal karyotypes in MDS patients with MF due to a combination of cytogenetics testing and FISH. Fu and colleagues indicated that monosomy 5 and 17 were significantly more frequent in a BMfibrosis group. ${ }^{13}$ However, we showed the most frequent karyotype of a typical abnormal karyotype in MDS patients was +8 . There was no significantly different distribution of abnormal karyotypes between MDS patients with mild/moderate MF and MDS cases without MF. However, in the previous studies, MDS patients with and without myelofibrosis have been reported differently in cytogenetics. Compared with cases with primary MDS without MF, MDS patients with moderate/severe MF show a higher frequency of cytogenetic abnormalities according to Della Porta and colleagues. ${ }^{5} \mathrm{Fu}$ and coworkers showed that MDS patients with or without MF have a similar cytogenetic risk distribution and frequency of an MK. ${ }^{1}$

In recent decades, moderate-to-severe myelofibrosis has been indicated as an adverse prognosis in patients with myelodysplastic syndromes in myelofibrosis proliferation neoplasms, ${ }^{6,21,22}$ therapy-related MDS and primary MDS, ${ }^{1}$ independent of age, performance status, and IPSS-R risk in multivariate analysis. ${ }^{6}$ We also revealed the independent prognostic importance of mild/moderate MF in MDS patients. In multivariate analyses, $\mathrm{MF}$ (mild/moderate) and the percentage of BM blasts had an independent negative impact on OS and PFS, as shown by Machherndl-Spandl and colleagues in MDS patients with severe MF. ${ }^{12}$ In primary MF BM fibrosis has been reported to be an adverse outcome even within the poor-risk group defined by the IPSS. ${ }^{14,23}$ However, in the long-term follow-up of MDS patients, we showed that mild/moderate MF played a vital part in the relatively low-risk group of IPSS-R, but had no obvious effect on MDS cases in the relatively high-risk group of IPSS-R. ${ }^{1}$ The reason may be due to the lower degree of fibrosis in MDS patients in our study due to the strict definitions in WHO2016C. More patients with severe MF were suspected of having MDS/myeloproliferative neoplasms or myeloproliferative neoplasms. Besides, consistent with the previous study, ${ }^{24}$ age also influences the clinical outcome of patients with MDS in our study. In our study, the MK was also an independent indicator of prognosis in MDS, as stated previously. ${ }^{11}$ Stratification of the MK subgroup demonstrated that MDS patients with mild/moderate MF were associated significantly with worse OS and PFS than those without MF in the long-term follow-up of the MK-negative subgroup. However, there were no differences in OS or PFS in MDS cases without MF in the MK-positive subgroup. Recent studies have shown that MKs are an additional adverse risk factor in MDS patients. ${ }^{11,25-27}$ The 
combination of MK and MF in MDS should be carefully considered when studying outcomes in MDS patients.

MDS patients with mild/moderate MF received more chemotherapy or decitabine and less supportive treatment compared with those without MF. To avoid the effect of treatment bias, we also undertook subgroup analyses for OS and PFS in MDS patients based on supportive treatment and nonsupportive treatment. There were no differences in the prognosis between MDS patients with or without mild/moderate MF in the non-supportive subgroup. However, in the supportive-treatment subgroup, MDS patients with mild/moderate MF were associated significantly with worse OS and PFS than those without MF.

We revealed the characteristics of MDS patients with MF, and the effects of mild/moderate MF on the prognosis of patients, but our study had some limitations. First, the number of patients with MF was relatively low because of the low incidence of MDS. Secondly, patient selection bias, geographic factors and ethnic differences should be reasons for the difference of our study with previous documents. Our study was in Chinese patients, which hampers the generalizability of our data. Thirdly, a few articles tested the gene mutation of MDS, ${ }^{28,29}$ but very small proportion of MDS patients did the gene mutation examination in our study. New, prospective, well-designed studies with a large cohort of MDS cases with mild/moderate MF are needed to confirm our findings.

\section{Conclusions}

Cytogenetics did not show a significant difference between MDS patients with and without mild/moderate MF. Mild/ moderate $\mathrm{MF}$ and $\mathrm{MK}$ were independent indicators of a poor clinical outcome in patients with MDS. Long-term follow-up revealed that $\mathrm{MDS}$ with mild/moderate MF could be a prognostic marker for MDS patients with a specific MK stratification and IPSS-R stratification.

\section{Funding}

This study was supported by grants from National Natural Science Foundation of China (81270598, 81473486, 81770210); Key Research and Development Program of Shandong Province (2018CXGC1213); Technology Development Projects of Shandong Province (2017GSF18189); Medicine and Health Science Technology Development Project of Shandong Province (2017WS200); Traditional Chinese Medicine Science and Technology Development Projects of Shandong Province (2019-0308), Science and Technology Development Program of Jinan (201907028); Taishan Scholar Foundation of Shandong
Province; Shandong Provincial Engineering Research Center of Lymphoma; Key Laboratory for Kidney Regeneration of Shandong Province; Academic promotion program of Shandong First Medical University; Shandong Governmentsponsored Study abroad program scholarship.

\section{Disclosure}

The authors declare no conflicts of interest in this work.

\section{References}

1. Fu B, Jaso JM, Sargent RL, et al. Bone marrow fibrosis in patients with primary myelodysplastic syndromes has prognostic value using current therapies and new risk stratification systems. Mod Pathol. 2014;27(5):681-689. doi:10.1038/modpathol.2013.187

2. Buesche G, Teoman H, Wilczak W, et al. Marrow fibrosis predicts early fatal marrow failure in patients with myelodysplastic syndromes. Leukemia. 2008;22(2):313-322. doi:10.1038/sj.leu.2405030

3. Lambertenghi-Deliliers G, Orazi A, Luksch R, Annaloro C, Soligo D. Myelodysplastic syndrome with increased marrow fibrosis: a distinct clinico-pathological entity. $B r J$ Haematol. 1991;78(2):161-166. doi:10.1111/j.1365-2141.1991.tb04411.x

4. Verhoef GE, de Wolf-peeters C, Ferrant A, et al. Myelodysplastic syndromes with bone marrow fibrosis: a myelodysplastic disorder with proliferative features. Ann Hematol. 1991;63(5):235-241. doi:10.1007/bf01698371

5. Della Porta MG, Malcovati L, Boveri E, et al. Clinical relevance of bone marrow fibrosis and CD34-positive cell clusters in primary myelodysplastic syndromes. J Clin Oncol. 2009;27(5):754-762. doi: $10.1200 / J C O .2008 .18 .2246$

6. Ramos F, Robledo C, Izquierdo-García FM, et al. Bone marrow fibrosis in myelodysplastic syndromes: a prospective evaluation including mutational analysis. Oncotarget. 2016;7(21):30492-30503. doi:10.18632/ oncotarget.9026

7. Arber DA, Orazi A, Hasserjian R, et al. The 2016 revision to the World Health Organization classification of myeloid neoplasms and acute leukemia. Blood. 2016;127(20):2391-2405. doi:10.1182/blood2016-03-643544

8. Thiele J, Kvasnicka HM, Facchetti F, Franco V, van der Walt J, Orazi A. European consensus on grading bone marrow fibrosis and assessment of cellularity. Haematologica. 2005;90 (8):1128-1132.

9. Simons A, Shaffer LG, Hastings RJ. Cytogenetic nomenclature: changes in the ISCN 2013 compared to the 2009 edition. Cytogenet Genome Res. 2013;141(1):1-6. doi:10.1159/000353118

10. Greenberg PL, Tuechler H, Schanz J, et al. Revised international prognostic scoring system for myelodysplastic syndromes. Blood. 2012;120(12):2454-2465. doi:10.1182/blood-2012-03-42 0489

11. Wang $\mathrm{N}, \mathrm{Xu} \mathrm{H}, \mathrm{Li} \mathrm{Q}$, et al. Monosomal karyotypes apart from complex karyotypes independently predict the outcome of myelodysplastic syndrome patients using a fluorescence in situ hybridization panel and conventional cytogenetics. Int J Lab Hematol. 2019;41 (4):519-529. doi:10.1111/ijlh.13041

12. Machherndl-Spandl S, Sega W, Bösmüller H, et al. Prognostic impact of blast cell counts in dysplastic bone marrow disorders (MDS and CMML I) with concomitant fibrosis. Ann Hematol. 2014;93 (1):57-64. doi:10.1007/s00277-013-1945-4

13. Fu B, Ok CY, Goswami M, et al. The clinical importance of moderate/severe bone marrow fibrosis in patients with therapy-related myelodysplastic syndromes. Ann Hematol. 2013;92(10):1335-1343. doi:10.1007/s00277-013-1776-3 
14. Scott BL, Storer BE, Greene JE, Hackman RC, Appelbaum FR, Deeg HJ. Marrow fibrosis as a risk factor for posttransplantation outcome in patients with advanced myelodysplastic syndrome or acute myeloid leukemia with multilineage dysplasia. Biol Blood Marrow Transplant. 2007;13(3):345-354. doi:10.1016/j.bbmt. 2006.10.030

15. Jaff MS, O'Briain DS. Excess of blood group B in primary myelofibrosis. Vox Sang. 1987;52(3):250-253. doi:10.1111/j.14230410.1987.tb03038.x

16. El Jellas K, Hoem D, Hagen KG, et al. Associations between ABO blood groups and pancreatic ductal adenocarcinoma: influence on resection status and survival. Cancer Med. 2017;6(7):1531-1540. doi:10.1002/cam4.1097

17. Xie J, Qureshi AA, Li Y, Han J, Chan A-W. ABO blood group and incidence of skin cancer. PLoS One. 2010;5(8):e11972. doi:10.1371/ journal.pone.0011972

18. Li Q, Wu T, Ma X-A, Jing L, Han L-L, Guo H. Prognostic role of ABO blood group in patients with unresectable hepatocellular carcinoma after transarterial chemoembolization. Ther Clin Risk Manag. 2018;14:991-998. doi:10.2147/TCRM.S160089

19. Rahbari NN, Bork U, Hinz U, et al. AB0 blood group and prognosis in patients with pancreatic cancer. BMC Cancer. 2012;12(1):319. doi:10.1186/1471-2407-12-319

20. Yamamoto F. Molecular genetics of the ABO histo-blood group system. Vox Sang. 1995;69(1):1-7. doi:10.1111/j.1423-0410.1995.tb00339.x

21. Petrova-Drus K, Chiu A, Margolskee E, et al. Bone marrow fibrosis in chronic myelomonocytic leukemia is associated with increased megakaryopoiesis, splenomegaly and with a shorter median time to disease progression. Oncotarget. 2017;8(61):103274-103282. doi:10.18632/oncotarget. 21870
22. Della Porta MG, Malcovati L. Myelodysplastic syndromes with bone marrow fibrosis. Haematologica. 2011;96(2):180-183. doi:10.3324/ haematol.2010.039875

23. Gianelli U, Vener C, Bossi A, et al. The European consensus on grading of bone marrow fibrosis allows a better prognostication of patients with primary myelofibrosis. Mod Pathol. 2012;25 (9):1193-1202. doi:10.1038/modpathol.2012.87

24. Balleari E, Salvetti C, Del Corso L, et al. Age and comorbidities deeply impact on clinical outcome of patients with myelodysplastic syndromes. Leuk Res. 2015;39(8):846-852. doi:10.1016/j.leukres.2015.05.007

25. Patnaik MM, Hanson CA, Hodnefield JM, Knudson R, van Dyke DL, Tefferi A. Monosomal karyotype in myelodysplastic syndromes, with or without monosomy 7 or 5 , is prognostically worse than an otherwise complex karyotype. Leukemia. 2011;25(2):266-270. doi:10.1038/ leu.2010.258

26. McQuilten ZK, Sundararajan V, Andrianopoulos N, et al. Monosomal karyotype predicts inferior survival independently of a complex karyotype in patients with myelodysplastic syndromes. Cancer. 2015;121(17):2892-2899. doi:10.1002/cncr.29396

27. Schanz J, Tüchler H, Solé F, et al. Monosomal karyotype in MDS: explaining the poor prognosis? Leukemia. 2013;27(10):1988-1995. doi:10.1038/leu.2013.187

28. Hussein K, Stucki-Koch A, Kreipe H. Profile of fibrosis-related gene transcripts and megakaryocytic changes in the bone marrow of myelodysplastic syndromes with fibrosis. Ann Hematol. 2018;97 (11):2099-2106. doi:10.1007/s00277-018-3411-9

29. Loghavi S, Al-Ibraheemi A, Zuo Z, et al. TP53 overexpression is an independent adverse prognostic factor in de novo myelodysplastic syndromes with fibrosis. $B r \quad J$ Haematol. 2015;171(1):91-99. doi:10.1111/bjh.13529

\section{Publish your work in this journal}

Cancer Management and Research is an international, peer-reviewed open access journal focusing on cancer research and the optimal use of preventative and integrated treatment interventions to achieve improved outcomes, enhanced survival and quality of life for the cancer patient.
The manuscript management system is completely online and includes a very quick and fair peer-review system, which is all easy to use. Visit http://www.dovepress.com/testimonials.php to read real quotes from published authors. 